\title{
Reproducing the Proximal Femur Anatomy Using Hip Resurfacing Implants
}

\author{
Julien Girard and Koen De Smet
}

\section{Key Points}

- Hip resurfacing (HR) is a personalized hip replacement procedure with restoration of biomechanical parameters with proximal femoral anatomy preservation.

- With HR, bone preservation is clearly an advantage on the femoral side.

- Hip joint stability allowing unrestricted range of motion with very low risk of dislocation.

- Possibility of returning to high-impact sports activities (running, football, judo, hockey, etc.).

- Better physiological restoration of spatial-temporal gait parameters versus standard head THA.

- Absence of thigh pain and optimal femoral loading.

- Preservation of hip joint proprioception.

- HR makes surgery easier in cases of femoral shaft deformity or when diaphysis implants are present.

J. Girard $(\bowtie)$

Orthopedics C Unit, Hopital Roger Salengro,

CHRU Lille, Lille Cedex, France

University of Lille, Lille, France

e-mail: julien.girard@chru-lille.fr

\section{K. De Smet}

Anca Medical Center, AMC, Xavier De Cocklaan 68.1, St Martens Latem Deurle, Belgium

\subsection{Why Perform Hip Resurfacing (Pros and Cons)?}

Today, we see younger patients with hip problems, so bone preservation and highly wearresistant bearings are becoming more relevant. Metal-on-metal hip resurfacing (HR) has now been used for 20 years. To achieve bone preservation, less bearing wear, and higher patient activity, the trade-off has been a more technically difficult surgery and subsequent failures. The lack of knowledge about HR implant design, tribology, and mechanical properties has led to a general desire to try out this concept but also to this procedure being abandoned.

In this respect, the biggest downsides of this procedure are that it cannot be performed in all hip cases and cannot be performed by every orthopedic surgeon. A minimum number of surgeries a year are becoming necessary to be allowed to perform HR surgery. Other drawbacks of metal-on-metal HR is that high bearing wear debris can cause adverse local tissue reactions (ALTR) or pseudotumors, and high amounts of cobalt and chromium ions are released systemically. General health problems have been linked to high cobalt levels in these cases but not in the normal functioning HR case. Even if we have a perfect design and perfect technique, this complication is difficult to avoid, as no hip joint surgery has a $100 \%$ success rate. Besides the expected numbers of failures, there 
is an unforeseen allergy problem, which can develop in $1 \%$ of females and $0.1 \%$ of males.

The well-known bone stock preservation on the femoral side, now also holds for the pelvic side, where no more bone is removed than with a total hip arthroplasty (THA), if the technique is done correctly. This was not the case in the beginning of the HR practice because of the learning curve and lack of large-diameter implants or thin cups. In case of revision surgery, it has been shown that if the patient and implant are monitored closely, revision can be done at the correct time, and the outcomes should not differ greatly, relative to primary THA. The increase in cup size after revision surgery is negligible and does not reflect the concerns raised in many papers [1].

Where other failures can be attributed to HR such as femoral neck fracture and loosening of the femoral head, the frequency has become very low in modern practice. There are more benefits to doing HR if all the expert recommendations and current practices are followed. Bone preservation and easier revisions are obvious, but many other benefits of HR have emerged in the last decade. Bone mineral density studies have shown that the bone stock returns to normal after HR.

We believe HR allows younger and more active patients to resume physical and sports activities without restriction. This difference has been demonstrated in an increasing number of randomized studies [2]. The risks of wear in active patients with metal-on-metal HR have been shown to have no influence, whereas the wear products and metal ions decline over time in a normal functioning HR [3]. Biomechanics and muscular moment arms are more easily restored to the normal native hip anatomy. Risk of dislocation in HR has always been low relative to THA and has become extremely rare.

The revision rate of HR depends on type of implant and its size; however, it has become clear that surgeon's experience has a major impact. Some authors see this as a negative. But in the right patient, like a young male patient with osteoarthritis, there is only a revi- sion burden of $9.5 \%$ at 16 years post HR versus $10.4 \%$ for THA in the same group based on registry data from the Australian Orthopedic Association National Joint Replacement Registry (AOANJRR 2017). Large volume/ single surgeon groups improve the survivorship up to $98 \%$ in this cohort.

A more unexpected finding is that patients with hip osteoarthritis undergoing metal-onmetal HR have reduced mortality in the long term compared to those undergoing cemented or uncemented THA. This difference persisted after extensive adjustment for confounding factors available in the retrieved data. Although residual confounding is possible, the observed effect size is large [4] (Fig. 4.1). These findings require further validation but are starting to be reported in several national hip registries. At present, after 20 years of experience with the new generation of metal-on-metal HR, we have separated the wheat from the chaff, and must continue to use proven designs with the correct technique and experience, in the correct patient.

\subsection{Clinical Evidence Supporting Hip Resurfacing}

Registries data: Outcomes of THA in younger patients ( $\leq 50$ years of age) are significantly worse compared to results in older patient groups. The 2016 Swedish Register found a cumulative survivorship in patients younger than 50 of $54.2 \%$ at 24 years' follow-up compared to $94.3 \%$ in patients older than 75 [5]. The 2016 AOANJRR indicated a cumulative percent revision of primary THA in patients aged less than 55 years of $8.5 \%$ and $12.7 \%$ at 10 and 15 years of follow-up, respectively [6]. On the other hand, HR in this specific population seems to work better. With the Birmingham Hip Resurfacing System (BHR), the 2016 National Joint Registry for England and Wales [7], the 2016 Australian Joint Registry [6], and the 2015 Swedish Registry [5] reported $90.1 \%$ survival at 12 years, $89.9 \%$ survival at 15 years, and $96.6 \%$ survival at 10 years, respectively. 

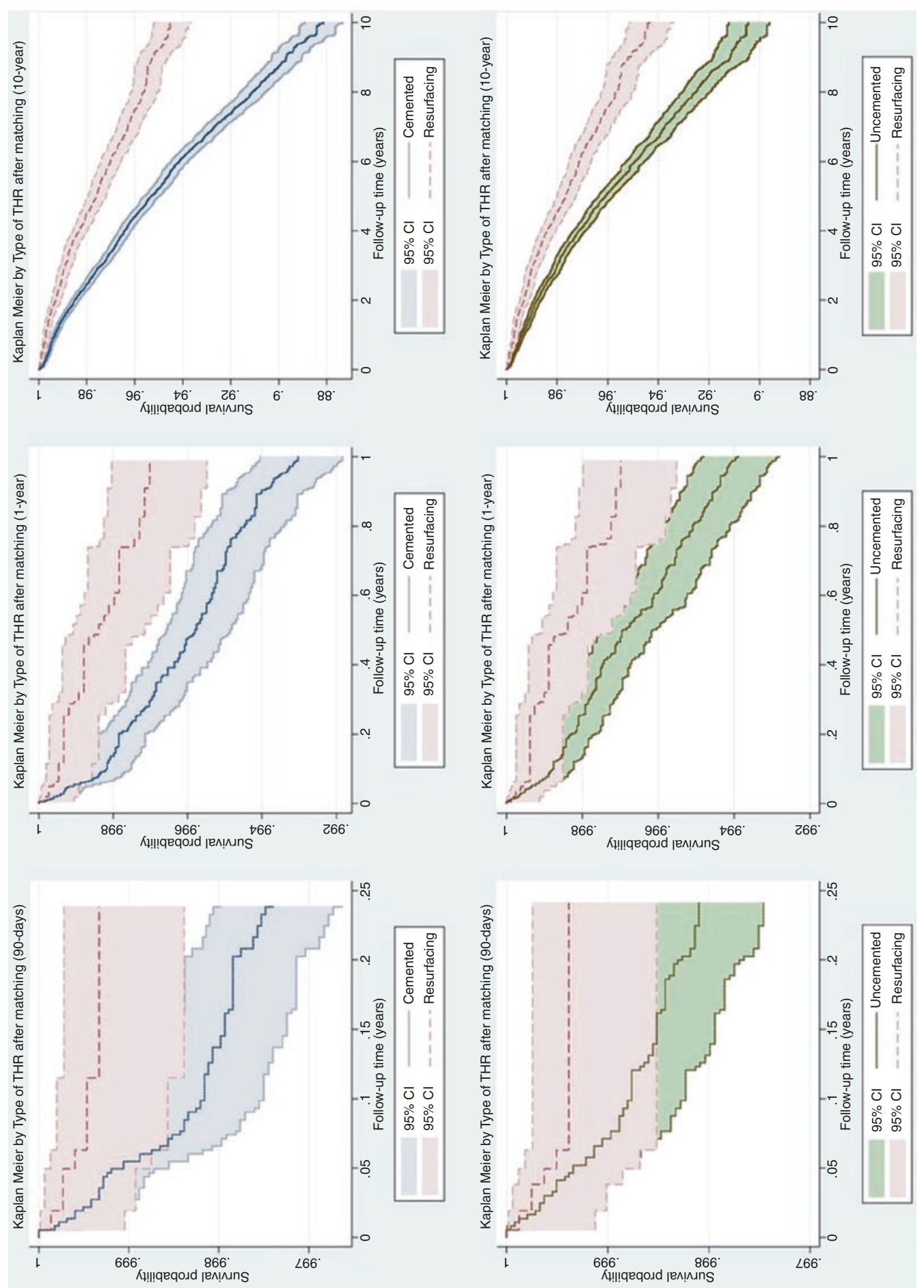

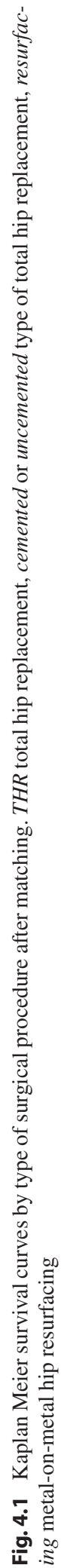


Recently, an international high-volume centers HR registry was created with patients $\leq 50$ years at surgery with a minimum of 3 years' follow-up $(11,386$ cases with a mean age of 42.7 years) [8]. There were $8459 \mathrm{HR}$ procedures in male patients (74.3\%) and 2926 in female patients $(25.7 \%)$ with a mean femoral head size of $49.7 \mathrm{~mm}$ and a mean follow-up time of 7.6 years (3 to 22). Overall survivorship was $89.1 \%$ at 22 years $(95 \%$ CI: $88.5-89.7 \%$ ). Survival was significantly superior in males- $92.7 \%$ at 21 years- $(95 \% \mathrm{CI}$ : $92.1-93.3 \%$ ) than in females-81.6\% at 22 years (95\% CI: 80.3-82.9\%).

Return to sports activities: Return to sport after hip arthroplasty is an increasingly common functional demand. However, there are few published studies on this subject and returning to high-impact sports appears to be challenging. HR seems to fulfill this functional demand since the prosthetic femoral head diameter is close to the native diameter, and the bearing has high wear resistance (without the risk of head fracture). Several studies have reported a high rate of return to low-, medium-, and high-impact sports after HR. To date, no international consensus recommendations exist on the possibility of returning to sports after hip arthroplasty. Nevertheless, HR allows a patient to resume physical and sports activities without restriction. The rate of return to sports after HR appears to be excellent. It is important to point out that no long-term studies have analyzed the impact of these activities on aseptic loosening. The most iconic example is with patients who participate in triathlons. Girard et al. [9] found rates of return to swimming, cycling, and running of 38/48 (79\%), $41 / 48$ (85\%), and 33/48 (69\%), respectively, in 48 Ironman-distance triathletes. More interesting, during the preoperative period, all patients had taken part in at least one Ironman competition and at 4.7 years of follow-up, 28/48 (58.3\%) had taken part in an Ironman competition with no decrease in their performance between the preoperative and postoperative periods.

Functional performance: The excellent hip function found after HR procedures is directly correlated with the conservative nature of the surgical procedure on the femoral head. With HR, the bone on the femoral bone side is preserved with two important effects: preservation of mechanoreceptors in the femoral neck and restoration of proximal femoral anatomy. Anatomical reconstruction after HR results in abductor and extensor moment arm preservation. In a prospective, randomized study comparing THA versus HR, biomechanical hip parameters were better restored with HR [10]. Leg length was restored to within $\pm 4 \mathrm{~mm}$ in $33(60 \%)$ of THA and $42(86 \%)$ of HR patients. Femoral offset was restored to within $\pm 4 \mathrm{~mm}$ in $14(25 \%)$ of THA and 29 (59\%) of HR cases. Beyond biomechanical restoration, gait analysis showed that in all planes of motion, HR restored the patient's normal gait pattern while THA required an adaptation. At 6 months and 2 years post surgery, THA patients had a lower walking speed compared to normal subjects and HR patients [11]. It could enhance center of mass control and increase energy generation during the push off phase. The same conclusion was drawn based on static and dynamic stabilometric analysis and postural coordination studies $[11,12]$. The advantage in terms of balance and postural control after HR results in better stability and motor patterns than observed after THA.

\subsection{Optimal Positioning of Hip Resurfacing Implants (Tricks and Tips)}

\subsubsection{What Are the Keys to Successful Hip Resurfacing?}

There are several keys to successful HR. The most important points are patient selection and appropriate surgical technique. Female patients have a greater risk of failure due to small femoral head size, high frequency of hip dysplasia, and potentially poor bone quality. Inflammatory disease, avascular necrosis, large femoral head cysts, and hip dysplasia appear to reduce survivorship. The best indication is primary osteoarthritis. Obesity is not a contraindication, but a minimum head diameter of $48 \mathrm{~mm}$ appears to be a prerequisite. 
The posterolateral approach is the "Queen of surgical approaches" for HR. Detaching the gluteus maximus tendon is unnecessary. Preserving soft tissues is important for vascularization and gluteal function. The external rotators must be cut 5-8 $\mathrm{mm}$ from the bone, preserving a small cuff. The capsule is cut at the level at the piriformis and not at the head-neck junction. Coagulation should not be performed at the head-neck junction. Preserving the capsule is key; we do not recommend performing a full $360^{\circ}$ capsulotomy.

Cup position is also crucial to the performance of metal-on-metal bearings. The cup should be positioned in $40^{\circ}$ inclination with anatomical anteversion. A steep cup amplifies the risk of increased metal ion levels and higher failure rate. On the other hand, a cup implanted in less than $30^{\circ}$ inclination can lead to impingement with the femoral neck in abduction and/or flexion. The transverse ligament is the key anatomical landmark. After impaction, the cup should be in line with the transverse ligament. This is the only prerequisite to impact the cup in an anatomical position and to avoid impingement. Preparing the femoral side first seems to be a smart option in order to optimize the acetabular exposure, to size the femoral neck perfectly and to achieve the optimal cup and femoral anteversion.

\subsubsection{Femoral Component Position}

It is very important to understand that the femoral neck is not circular in shape. Usually, it has more of an ovoid shape. The second important point is the definition of head-neck offset: distance between the head equator and femoral neck surface. Given that the femoral neck is not circular, this offset is not constant around the head/neck circumference. Third, HR has the worse headneck offset of all the hip implant designs. After hip resurfacing, the femoral head-neck diameter offset is lower than conventional THA. In fact, the head-neck diameter ratio after conventional THA is close to 2 (assuming a 28 -mm-diameter head and 12/14-mm-diameter neck), more than 3 for large-diameter heads, and around 1.2 for HR. This point is crucial. The risk of cam effect with impingement between the femoral neck and the cup or acetabular bone is one of the modes of failure of the HR. So correct component positioning is crucial and intraoperative testing is essential. The position of the cup and femoral components is interrelated, and excessive cup anteversion inexorably leads to a posterior cam effect. A retroverted cup leads to an anterior cam and an overly inclined cup to a superior cam.

In the same way, a low head-neck diameter offset may be detrimental to achieving better hip flexion. After conventional THA, range of motion is limited by "implant to implant" cam effect while with HR, it is limited by "cup component to femoral neck bone" contact. Hip flexion is the most important motion for daily activities. Maximum anterior head-neck offset is necessary to avoid cup-bone contact and increase the degree of flexion at which it occurs (Fig. 4.2). Anterior translation of the femoral component relative to the central femoral neck axis may improve anterior head-neck offset and hip flexion. Usually, the position of the femoral component is flush with the posterior cortex of the femoral neck. Considering that $1 \mathrm{~mm}$ of anterior offset increases hip flexion by $5^{\circ}$, anterior translation of the femoral component appears to be an attractive way to increase range of motion [13]. But anterior head-neck offset is very sensitive, and it is important to avoid drastically reducing the posterior offset.

HR is a surgical compromise. Each time the femoral position is optimized, the opposite position may be compromised. So, improving hip

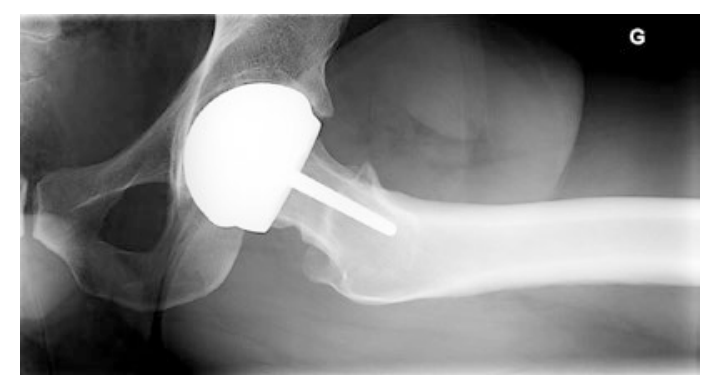

Fig. 4.2 Optimal femoral position. The femoral component is parallel to the neck with physiological anteversion. Notice the slight shift from posterior to anterior which led to better anterior head-neck offset 
flexion by increasing anterior translation should be done carefully to avoid reducing the range of motion in the opposite direction. In fact, a completely symmetrical position of the femoral cup is not the rule. To summarize, more flexion than extension is required for daily activities. A posterior-to-anterior shift seems to be the most attractive option to improve range of motion. Removing osteophytes at head-neck junction can be done with caution after femoral impaction.

The last point in the offset femoral position is the femoral metallic offset of each implant. It reaches 3-4 $\mathrm{mm}$ and the cement mantle thickness adds $0-1.5 \mathrm{~mm}$. Therefore, HR femoral headneck offset varies from 3 to $5 \mathrm{~mm}$. Other tricks can be used to increase range of motion:

- Careful anterior femoral osteoplasty can improve anterior offset and decrease the risk of a cam effect. But surgeons must be aware of the risk of neck fracture if the osteoplasty crosses the neck cortex.

- Acetabular rim osteophyte removal is essential. A 2-3 mm width of acetabular bone must be preserved on the anterior wall to avoid the risk of iliopsoas impingement. But if necessary, acetabular bone should be cleared around the cup.

- Modifying the femoral stem angle is not recommended. In fact, retroversion of the femoral component has little influence on the cam effect and leads to contact between the stem and neck.

- Increasing the implants' diameter could theoretically increase the femoral-head offset. But the risk of groin pain, acetabular bone fracture, and psoas irritation do not allow insertion of large cups. Moreover, the femoral component must be fitted on the bony femoral head without any defect.

A slight valgus placement of the femoral component leads to better biomechanical performance. A valgus of $5^{\circ}$ to $10^{\circ}$ compared to the native femoral neck is recommended [14]. A varus position exposes to neck a stress raiser while excessive valgus could produce a superior notch. Two useful intraoperative landmarks are the inferior part of the femoral neck and the femoral head fovea. The femoral K-wire should be placed in a slight valgus position relative to the inferior femoral neck line and about $1 \mathrm{~cm}$ higher than the fovea.

To achieve the optimal hip range of motion and avoid femoral neck impingement on the cup component, the goal is to reproduce the natural femoral head-neck offset around the entire femoral neck. That means the offset could be modified for each deformity (Legg-CalvePerthes, post traumatic...). This is very different to conventional THA where the proximal femur is first resected and then reconstructed with a femoral stem. The stem should reproduce patient anatomy, biomechanical properties, and restore soft tissue tension. HR is an anatomypreserving surgical procedure that keeps the proximal femur and minimizes anatomical distortion. With THA, surgeons have many implant options: multiple stem sizes, prosthetic head modularity, different stem neck-shaft angles, standard or high offset stems, different head diameters, anti-dislocation lips, etc. With HR, none of these possibilities exist and preserving the proximal femoral anatomy leads to exact biomechanical reconstruction of the joint (well described in randomized study). In fact, with THA, the biomechanical restoration is correlated with stem fixation. If stem stability is suboptimal, it could lead to implant over-sizing and leg overlengthening and increase the femoral offset. With HR, the femoral component diameter is close to the native head diameter. The stability of the prosthetic head is immediate and optimal and under-sizing is impossible.

At the end of the procedure, the ability to view the position of both components is very valuable. In case of primary osteoarthritis without neck deformity and with a standard stem-shaft angle, the two components must be parallel to each other with the hip in neutral position (no rotation, no abduction, leg in line with trunk). This means the femoral component is in slight valgus $\left(140^{\circ}\right)$ and the cup is near $40^{\circ}$ inclination. This point is crucial because it helps to avoid impingement between the neck and cup. The last check is performed to detect potential cam impingement 
(anterior or posterior). An acetabular rim osteoplasty or femoroplasty can be done at this point if needed.

\subsubsection{Vascularization}

Thorough knowledge of the vascular anatomy of the femoral head is necessary before starting a HR procedure. The retinacular vessels along the posterolateral and inferomedial femoral neck must be located. Throughout the procedure, the retinacular vessels should be preserved as well as all the soft tissues around the femoral head. Maintaining the blood supply to the femoral neck is vital. On the other hand, the posterior approach that inevitably disrupts the main blood supply is commonly used for HR. But the large majority of studies do not report head collapse or heat-induced bone necrosis. The blood supply of an arthritic femoral head can come from intraosseous vessels rather than from retinacular vessels. Moreover, some vascular anastomoses between the femoral epiphysis and metaphysis could increase the neck's blood supply. But the surgeon should be aware that these two possibilities do not mean the retinacular vessels do not need to be preserved.

Because of blood supply vulnerability during posterolateral approach, other approaches have been investigated. The main goal of each one is femoral head vascularization. These approaches are the direct lateral approach, anterior approach, and trochanteric flip approach. To date, none of these surgical approaches have been shown to lower the rate of head collapse. The most attractive surgical approach is still the posterolateral approach but with minimal soft tissue disruption: no release of the gluteus maximus extension, no circumferential capsulotomy, no release of the gluteus medius on the iliac bone and preservation of reticular vessels and soft tissues along the neck.

\subsubsection{Femoral Cementing Technique}

The femoral cementing technique is an important factor for long-term HR survival. Additional drill holes should be made in the prepared femoral head to increase the fixation area. A distance of at least $1 \mathrm{~cm}$ is required between cement holes to avoid thermal osteonecrosis. Five to ten anchoring holes $7 \mathrm{~mm}$ in depth and $4 \mathrm{~mm}$ in diameter are preferred. Some surgeons recommended placing a suction device into the lesser trochanter with femoral head pulse lavage in order to optimize cement penetration. However, this could lead to deep cement penetration and subsequently thermal necrosis. A dome hole seems sufficient before applying low viscosity cement. The merits of two cement application techniques-indirect filling with cement into the component or direct cement packing on the femoral head-continue to be debated. It is important to note that the cement mantle and penetration depth vary greatly depending on cement viscosity, head bone density, clearance between the reamed head and femoral component, and implant design.

\subsection{Future Developments in Hip Resurfacing}

There is an alternative type of surgery known as HR, which is carried out on younger patients. Unlike THA, the surgeon only removes the diseased cartilage from the hip joint and resurfaces it using a metal-on-metal implant. However, in some patients, the metal particles released by the implant cause tissue reactions with clinical implications. Because of the failures related to metallosis, the concern about metal ions and the risk of metal allergy has led to new developments, especially for female patients, who tend to have a smaller head size and higher percentage of allergy. While the need for surgeons to have substantial experience with the technique remains, some new developments are ready for the orthopedic joint market.

A new resurfacing implant with polyethylene cup is being tested, whereas ceramic-on-ceramic resurfacing looks to be a logical design for resurfacing implants. No matter what is produced or engineered, we should be aware there can be snags and unexpected problems can develop. Squeaking with ceramic-on-ceramic is a well-known problem 
in THA. The reported incidence of noisy ceramicon-ceramic hips ranges from $1 \%$ to $29 \%$ depending on how the "noise" is defined [15]. Some acoustic studies have distinguished between squeaking and other types of noise such as clicking, clunking, popping, and grating in metal-on-metal resurfacing. The question remains whether these could appear in ceramic-on-ceramic resurfacing bearings. Squeaking in large-diameter metal-on-metal hip replacements has been associated with increased clearance and reduced lubrification [16]. In the newer ceramic-on-ceramic large-diameter total hips (head diameters up to $48 \mathrm{~mm}$ ), the squeaking rate increases with head diameter ( $36 \mathrm{~mm}$ to $48 \mathrm{~mm}$ ) [17]. These well-documented THA findings need to be addressed and documented during clinical trials of all new ceramic-on-ceramic resurfacing bearings coming on the market [18]. Ceramic fractures due to high impact should be a smaller concern based on stress tests done in the laboratory. Hopefully, resurfacing will not reproduce these complications, but we have to be aware that new problems can occur, like the trunnionosis problem with large metal heads on a stem in THA - a problem we never experienced in 60 years of joint surgery!

The custom polyethylene hip resurfacing was designed by pioneering orthopedic surgeon Derek McMinn. It is an alternative to patients with metal allergies. The cup is made from highly cross-linked polyethylene and has a layer of titanium porous coating on the outer surface, like the RM Pressfit cup (Matthys $\rightarrow$ ) with a mean survival rate of $94.4 \%$ for aseptic loosening after 20 years. Dr. Pritchett (Seattle, USA) has produced Synovo Preserve implants made with cross-linked polyethylene which is stronger, lighter, and more wear resistant than conventional polyethylene. Both designs use a cobalt-chrome head, thus there still is a theoretical risk of allergy, just like in knee implants. But the fact these are hard-on-soft bearings that will not last a lifetime in younger active patients does not make them the ideal new HR development.

Ceramic-on-ceramic HR appears to be a better idea for reducing the risk of wear and allergy (Fig. 4.3). Justin Cobb at the Imperial College London was the first surgeon in the world to resurface patients' hips with ceramic-on-ceramic implants. A clinical trial has been designed to

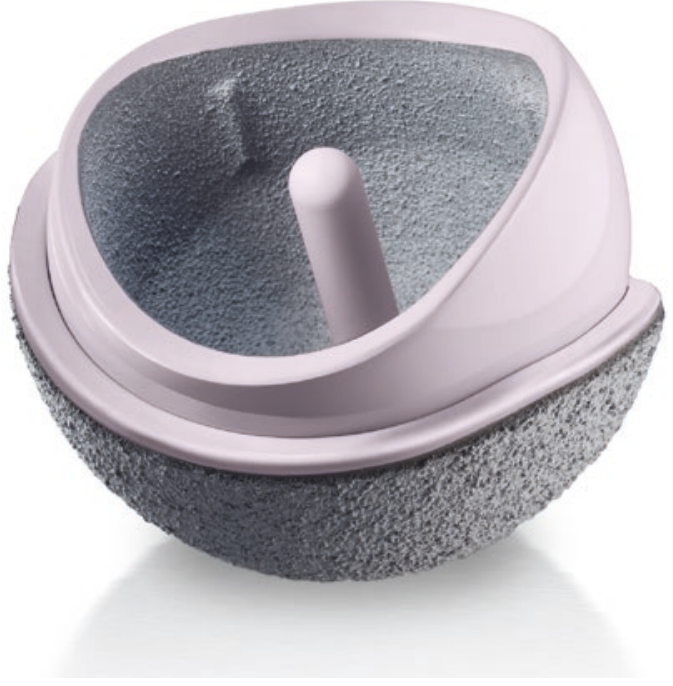

Fig. 4.3 Ceramic-on-ceramic $\mathrm{H} 1{ }^{\circledR}$ hip resurfacing components (Embody, London, UK) first implanted by Pr. Justin Cobb in 2017

show ceramic implants are suitable for both men and women, as conventional HR techniques are currently less suitable for female patients. The new device, called "H1" (Fig. 4.3), has a contoured cup and $\mathrm{BIOLOX}^{\rightarrow}$ delta on $\mathrm{BIOLOX}^{\rightarrow}$ delta bearing. The contoured design is designed to better match the patient's anatomy and prevent impingement. The cup has a titanium porous coating, and the head is not cemented. It is important to realize that such designs are completely new; thus, unexpected problems may develop. They should be evaluated for a long time before they are made fully available to the orthopedic market. The same is true for the new ReCerf ${ }^{\mathrm{TM}}$ Hip Resurfacing Arthroplasty from MatOrtho $\rightarrow$ which uses ceramic monoblock components by Ceramtec $\rightarrow$-femoral heads and acetabular cups-with no metal components.

Other companies are working on new HR designs with other bearing options. History always comes back to the resurfacing technique because it looks like a more anatomical, biomechanical, and logical treatment. From Charnley's soft Teflon bearing in the 1950s, to the Haboush (US) metal-on-metal bearing in 1953, to the 1970s with Gerard (France) and Muller (Switzerland) and the Wagner prosthesis in the 1980s, resurfacing will always remain an option. Today, there is extensive history with metal-on-metal resurfac- 
ing, with experimental work done than in typical THA implants. It is vital that we do not make the same mistakes twice, and we should be wary of any newly introduced solution that is inadequate at this moment.

\subsection{Why Do We Recommend Hip Resurfacing? (Convincing Arguments)}

The main reasons I recommend HR rather than THA for younger patients are:

- Bone preservation: With HR, bone preservation is clearly an advantage on the femoral side. Moreover, femoral neck bone density increases postoperatively due to physiological loading.

- No dislocation: In a randomized controlled trial, Vendittoli and al. [10] reported a 0\% dislocation rate in the HR group compared to $3 \%$ in the THA group. Pollard et al. [19] reported a dislocation rate of $7.4 \%$ among 54 THA patients while none occurred in a group of 54 HR patients.

- Possibility of returning to high-impact sports activities (running, football, judo, hockey, etc.).

- Physiological restoration of spatial-temporal gait parameters.

- Restoration of biomechanical parameters: No leg length discrepancy and normal femoral offset are possible after HR.

- Absence of thigh pain.

- Optimal femoral loading.

- Preservation of hip joint proprioception.

- Possibility of performing HR even in cases of femoral shaft deformity or when existing implants cannot be removed.

\section{References}

1. De Smet K, Van Der Straeten C, Van Orsouw M, Doubi R, Backers K, Grammatopoulos G. Revisions of metal-on-metal hip resurfacing: lessons learned and improved outcome. Orthop Clin N Am. 2011;42(2):259-69.
2. Lavigne M, Masse V, Girard J, Roy AG, Vendittoli PA. Return to sport after hip resurfacing or total hip arthroplasty: a randomized study. Rev Chir Orthop Reparatrice Appar Mot. 2008;94(4): 361-7.

3. Van Der Straeten C, Van Quickenborne D, De Roest B, Calistri A, Victor J, De Smet K. Metal ion levels from well-functioning Birmingham hip resurfacings decline significantly at ten years. Bone Joint J. 2013;95-B(10):1332-8.

4. Kendal AR, Prieto-Alhambra D, Arden NK, Carr A, Judge A. Mortality rates at 10 years after metalon-metal hip resurfacing compared with total hip replacement in England: retrospective cohort analysis of hospital episode statistics. BMJ. 2013;347:f6549. https://doi.org/10.1136/bmj.f6549.

5. Swedish Register. https://registercentrum.blob. core.windows.net/shpr/r/Annual-Report-2015H19dFINOW.pdf20.

6. Australian Orthopedic Association National Joint Replacement Registry. https://aoanjrr.sahmri.com/fr/ annual-reports-2016.

7. 2016 National Joint Register of England and Wales. http://www.njrcentre.org.uk/njrcentre/NewsandEvents/NJR14thAnnualReportrecordnumberofproceduresduring201617/tabid/1453/Default.aspx.

8. Van Der Straeten C. Results from a worldwide HR data base. Seoul: International Society for Technology in Arthroplasty; 2017.

9. Girard J, Lons A, Pommepuy T, Isida R, Benad K, Putman S. High-impact sport after hip resurfacing: The Ironman triathlon. Orthop Traumatol Surg Res. 2017;103(5):675-8.

10. Vendittoli PA, Ganapathi M, Roy AG, Lusignan D, Lavigne M. A comparison of clinical results of hip resurfacing arthroplasty and $28 \mathrm{~mm}$ metal on metal total hip arthroplasty: a randomised trial with 3-6 years follow-up. Hip Int. 2010;20(1):1-13.

11. Szymanski C, Thouvarecq R, Dujardin F, Migaud H, Maynou C, Girard J. Functional performance after hip resurfacing or total hip replacement: a comparative assessment with non-operated subjects. Orthop Traumatol Surg Res. 2012;98(1):1-7.

12. Bouffard V, Nantel J, Therrien M, Vendittoli PA, Lavigne M, Prince F. Center of mass compensation during gait in hip arthroplasty patients: comparison between large diameter head total hip arthroplasty and hip resurfacing. Rehabil Res Pract. 2011;2011:586412.

13. Girard J, Krantz N, Bocquet D, Wavreille G, Migaud H. Femoral head to neck offset after hip resurfacing is critical for range of motion. Clin Biomech (Bristol, Avon). 2012;27(2):165-9.

14. Beaulé PE, Harvey N, Zaragoza E, Le Duff MJ, Dorey FJ. The femoral head/neck offset and hip resurfacing. J Bone Joint Surg Br. 2007;89(1):9-15.

15. Keurentjes, et al. High incidence of squeaking in THAs with alumina ceramic-on-ceramic bearings. Clin Orthop Relat Res. 2008;466(6): 1438-43. 
16. Brocket, et al. The influence of clearance on friction, lubrication and squeaking in large diameter metalon-metal hip replacements. J Mater Sci Mater Med. 2008;19(4):1575-9.

17. Blakeney WG, Beaulieu Y, Puliero B, Lavigne M, Roy A, Massé V, Vendittoli PA. Excellent results of large-diameter ceramic-on-ceramic bearings in total hip arthroplasty. Bone Joint J. 2018;100-B(11): 1434-41.
18. Tai S, et al. Squeaking in large diameter ceramicon-ceramic bearings in total hip arthroplasty. $\mathrm{J}$ Arthroplast. 2014;30(2) https://doi.org/10.1016/j. arth.2014.09.010.

19. Pollard TC, Baker RP, Eastaugh-Waring SJ, Bannister GC. Treatment of the young active patient with osteoarthritis of the hip. A five- to seven-year comparison of hybrid total hip arthroplasty and metal-on-metal resurfacing. J Bone Joint Surg Br. 2006;88(5):592-600.

Open Access This chapter is licensed under the terms of the Creative Commons Attribution 4.0 International License (http://creativecommons.org/licenses/by/4.0/), which permits use, sharing, adaptation, distribution and reproduction in any medium or format, as long as you give appropriate credit to the original author(s) and the source, provide a link to the Creative Commons license and indicate if changes were made.

The images or other third party material in this chapter are included in the chapter's Creative Commons license, unless indicated otherwise in a credit line to the material. If material is not included in the chapter's Creative Commons license and your intended use is not permitted by statutory regulation or exceeds the permitted use, you will need to obtain permission directly from the copyright holder. 